\title{
Evolution of a gastric carcinoma cell-specific DNA aptamer by live cell-SELEX
}

\author{
HONG-YONG CAO ${ }^{1,2}$, AI-HUA YUAN ${ }^{1}$, XUE-SONG SHI $^{1}$, WEI CHEN ${ }^{1}$ and YI MIAO ${ }^{2}$ \\ ${ }^{1}$ Department of General Surgery, Nanjing Hospital Affiliated to Nanjing Medical University; ${ }^{2}$ Department \\ of General Surgery, First Affiliated Hospital of Nanjing Medical University, Nanjing, Jiangsu, P.R. China
}

Received April 13, 2014; Accepted June 12, 2014

DOI: 10.3892/or.2014.3411

\begin{abstract}
Aptamers have emerged as promising molecular probes for disease diagnosis and therapy. In the present study, the entire live cell-SELEX method was used to generate gastric cancer cell-specific aptamers. Human gastric carcinoma AGS cells were used as target cells for positive selections and human normal gastric epithelial GES-1 cells as the negative cells for counter selections. The selection procedure was monitored by gel electrophoresis and flow cytometric analysis. By successive in vitro evolutions and subsequent cloning and sequencing, a gastric carcinoma cell-specific DNA aptamer termed cy-apt 20 with minimal recognition to the controls was identified from the final enriched ssDNA pool. Flow cytometry binding assays revealed that cy-apt 20 had a $>70 \%$ binding rate to AGS cells and $<30 \%$ binding affinity to non-gastric cancer cells. Furthermore, the targeting recognition of AGS cells was established by using minimal doses of FITC-cy-apt 20 that continued for a long period of time. As visualized by fluorescence imaging, the majority of AGS cells were stained by FITC-cy-apt 20. The fluorescence intensity of AGS cells was $\sim 6$-fold over that of non-AGS cells. The present study demonstrated that the entire live cell-SELEX was simple, but effective in generating gastric cancer cell-specific aptamers, and that the aptamer cy-apt 20 has great potential to be used for the study and diagnosis of gastric cancer.
\end{abstract}

\section{Introduction}

Disease biomarkers are widely used in medicine, but few biomarkers are useful for the diagnosis and monitoring of gastric cancer. The expansion of new technologies for molecular diagnostics and tumor-targeted therapy also increased the need to develop highly specific targeting ligands for mole-

Correspondence to: Professor Yi Miao, Department of General Surgery, First Affiliated Hospital of Nanjing Medical University, 300 Guangzhou Road, Nanjing, Jiangsu 210029, P.R. China E-mail: njmuyah@163.com

Key words: DNA aptamer, molecular probe, live cell-SELEX, fluorescence imaging, gastric cancer cules that are differentially expressed in gastric cancer cells and normal tissues (1). Gastric cancer is an aggressive disease often diagnosed at an advanced stage. Despite the improvements in surgical and adjuvant treatment, gastric cancer remains a global public health problem with a 5 -year survival of $<25 \%(2,3)$. In the past two decades, new biomarkers have been identified with the potential to ameliorate the diagnosis and treatment of gastric cancer. However, initially promising biomarkers have not been validated for clinical use. The main challenge in identifying reliable predictive biomarkers is individual genetic variation and tumor heterogeneity, which have yet to be adequately determined. Biomarkers currently used in the clinic such the carcinoembryonic antigen (CEA) and carbohydrate antigen CA72-4 and CA19-9 are, not only elevated at late stages of cancer development, but also expressed by other types of cancer and even by non-cancerous tissues, providing limited information $(1,4)$. Thus, more reliable prognostic and predictive biomarkers may be extremely valuable for diagnosing, stratifying, and targeting gastric cancer and ultimately, improving patient survival.

Recently, a new class of molecular probes termed aptamers has emerged as promising molecular probes for disease diagnosis and therapy (5). Aptamers are single-stranded DNA (ssDNA), RNA or modified nucleic acids that bind specifically to targets, which range from small organic molecules to proteins (6). The basis for target recognition is the tertiary structures formed by the single-stranded oligonucleotides (7). Aptamers possess numerous advantageous characteristics, including small size, lack of immunogenicity, easy and reproducible synthesis, high-binding affinity and molecular specificity, fast tissue penetration and low toxicity, tenability in binding affinity and long-term stability $(5,6)$. Aptamers are generated by an iterative in vitro evolution procedure known as systematic evolution of ligands by exponential enrichment (SELEX) $(8,9)$. This method has been extended to develop a live cell-based SELEX (live cell-SELEX) for generating cancer cell-specific aptamers (10). Accumulating evidence has demonstrated that the live cell-SELEX is simple, fast, straightforward, reproducible and most importantly, effective even when there is only a minor difference between a cancer cell and an untransformed cell of the same type of tissue $(5,6,11)$. Using cell-SELEX, a group of aptamers that specifically recognize leukemia cells $(12,13)$, breast $(14)$, lung (15) and colorectal cancer cells (16) were generated. 
Results of those studies suggested that the live cell-SELEX is a promising strategy for identifying biomarkers for gastric cancer diagnosis and targeting therapy.

In the present study, a specific DNA aptamer against gastric carcinoma cells was selected using live cell-SELEX. The aptamer was confirmed by a series of experiments as having the potential to be used for the study and diagnosis of gastric cancer.

\section{Materials and methods}

Cell lines and cell culture. Human normal gastric epithelial GES-1, gastric carcinoma AGS, HepG2 liver hepatoma and SW620 colon carcinoma cell lines were obtained from the American Type Culture Collection (ATCC; Manassas, VA, USA). GES-1, AGS and SW620 cells were maintained and propagated in Dulbecco's minimal essential medium (DMEM) supplemented with $20 \%$ fetal bovine serum (FBS) (both from HyClone, Logan, UT, USA) and $100 \mathrm{U} / \mathrm{ml}$ penicillin-streptomycin (Sigma, St. Louis, MO, USA). HepG2 cells were maintained and propagated in RPMI-1640 (Sigma) supplemented with $10 \%$ FBS and $100 \mathrm{U} / \mathrm{ml}$ penicillin-streptomycin. The cells were cultured in $100 \times 20-\mathrm{mm}$ culture dishes at $37^{\circ} \mathrm{C}$ in a humidified atmosphere containing $5 \% \mathrm{CO}_{2}$. The experiments were carried out using cultures that had reached $>90 \%$ confluence.

DNA primers and libraries. Random DNA primers and a library were designed using the Integrated DNA Technologies software (IDT, Coralville, IA, USA), synthesized by standard phosphoramidite chemistry with an automated DNA synthesizer (3400 DNA Synthesizer; Applied Biosystems Inc., Foster City, CA, USA) and purified by reversed-phase high-performance liquid chromatography (RP-HPLC; Shanghai Sangon Biological Company, Shanghai, China). The purified library contained a central randomized sequence of 52 nucleotides (nt) flanked by two 18-nt primer hybridization sites (ATACCAGCTTATTCAATT-52-ntAGATAGTAAGTGCAATCT). The forward and reverse primers used in the PCR performed in the process of cell-SELEX were separately labeled with fluorescein isothiocyanate: (FITC), (5'-FITC-ATACCAGCTTATTCAATT-3') and biotin (Bio), (5'-Bio-AGATAGTAAGTGCAATCT-3') at the $5^{\prime}$ end in order to synthesize double-labeled and double-stranded DNA molecules.

Procedure of cell-SELEX. AGS cells were used as target cells for the positive selections and GES-1 as negative cells for the counter selections. Prior to the selection, culture cells were harvested by non-enzymatic cell dissociation solution and then washed twice with washing buffer $(4.5 \mathrm{~g} / 1$ glucose and $5 \mathrm{mM} \mathrm{MgCl}_{2}$ in Dulbecco's phosphate-buffered saline with calcium chloride and magnesium chloride) (both from Sigma). A total of 200 pmol library or DNA pool was dissolved in $400 \mu \mathrm{l}$ of binding buffer (500 nM/l). The binding buffer was prepared by adding $0.1 \mathrm{mg} / \mathrm{ml} \mathrm{tRNA}$ and $1 \mathrm{mg} / \mathrm{ml}$ of bovine serum albumin (BSA) (both from Sigma) into the washing buffer. The library or DNA pool was denatured at $95^{\circ} \mathrm{C}$ for $5 \mathrm{~min}$ and quickly cooled on ice for $10 \mathrm{~min}$. The DNA pool was then incubated with $5 \times 10^{6}$ AGS cells at $4^{\circ} \mathrm{C}$ on a shaker for $40 \mathrm{~min}$. Following incubation, the cells were washed three times with washing buffer to remove unbound sequences. The cell-DNA complex was resuspended in $400 \mu \mathrm{l}$ binding buffer and heated at $95^{\circ} \mathrm{C}$ for $15 \mathrm{~min}$ and centrifuged at 14,000 rpm to elute the bound DNAs. The eluted DNAs were incubated with $1 \times 10^{7} \mathrm{GES}-1$ cells for counter selection at $4^{\circ} \mathrm{C}$ on a shaker for $40 \mathrm{~min}$. The cells were subsequently centrifuged at $14,000 \mathrm{rpm}$ for $5 \mathrm{~min}$. The supernatant containing the ssDNA was recovered and amplified by polymerase chain reaction (PCR) using FITC- and biotin-labeled primers. Amplifications were carried out in an Eppendorf PCR Thermocycler (Eppendorf GAC 22331; Hamburg, Germany). The PCR amplification conditions of the primers and libraries were optimized prior to the selections. The reaction system consisted of $50 \mathrm{mM} \mathrm{KCl}$, $10 \mathrm{mM}$ Tris- $\mathrm{HCl}$ (pH 8.3), $1.5 \mathrm{mM} \mathrm{MgCl}_{2}, 25 \mathrm{mM}$ dNTPs (Takara), $0.5 \mathrm{mM}$ of each primer, and $5 \mathrm{U} / \mathrm{ml}$ Hot start Taq DNA Polymerase (Takara). The amplification steps included 35 cycles of $3 \mathrm{~min}$ at $95^{\circ} \mathrm{C}, 45 \mathrm{sec}$ at $94^{\circ} \mathrm{C}, 30 \mathrm{sec}$ at $63.5^{\circ} \mathrm{C}$ and $30 \mathrm{sec}$ at $72^{\circ} \mathrm{C}$, followed by the final $10 \mathrm{~min}$ of extension at $72^{\circ} \mathrm{C}$. The selected sense ssDNA strands were separated from the biotinylated antisense ssDNA strands by alkaline denaturation and purified by streptavidin-coated sepharose beads (Amersham Biosciences). The selected ssDNA was then dried and resuspended in binding buffer for the subsequent round of selection. After 12 rounds of selections, the final selected ssDNA pool was PCR-amplified and cloned into Escherichia coli using the TOTO TA cloning kit (Invitrogen, Carlsbad, CA, USA). Cloning of the PCR products and sequencing of the selected sense ssDNA were performed by the Shanghai Sangon Biological Company.

Gel electrophoresis of PCR products. To confirm the PCR products of each round of selection containing the selected ssDNA strands from the library, agarose gel electrophoresis was routinely performed. The nucleic acid content was first determined at $260 \mathrm{~nm}$ using a UV spectrophotometer (BioPhotometer; Eppendorf, Germany) and then monitored by electrophoresis on a $2 \%$ agarose gel and visualized by ethidium bromide staining. The correct molecular weight was confirmed by a DNA molecular weight marker (Boehringer, Mannheim, Germany).

Flow cytometric analysis. To assess the enrichment of specific aptamer candidates and the binding capacity and affinity of the selected aptamer candidates to AGS, cells were cultured at $90 \%$ confluence were harvested by non-enzymatic cell dissociation solution and then washed twice with washing buffer. Cells $\left(5 \times 10^{5}\right)$ were incubated with varying concentrations of FITC-labeled selected ssDNA in $200 \mu \mathrm{l}$ binding buffer on ice for various time lengths. Cells were then washed twice with washing buffer and suspended in $200 \mu \mathrm{l}$ washing buffer. The fluorescence was analyzed by flow cytometry (BD FACSCalibur; BD Biosciences). The FITC-labeled unselected ssDNA to AGS and the FITC-labeled selected ssDNA to GES, HepG2 and SW620 were used as controls. All the experiments were repeated three times. The mean fluorescence intensity of target cells labeled by selected ssDNA was calculated by subtracting the mean fluorescence intensity produced by unselected ssDNA. 

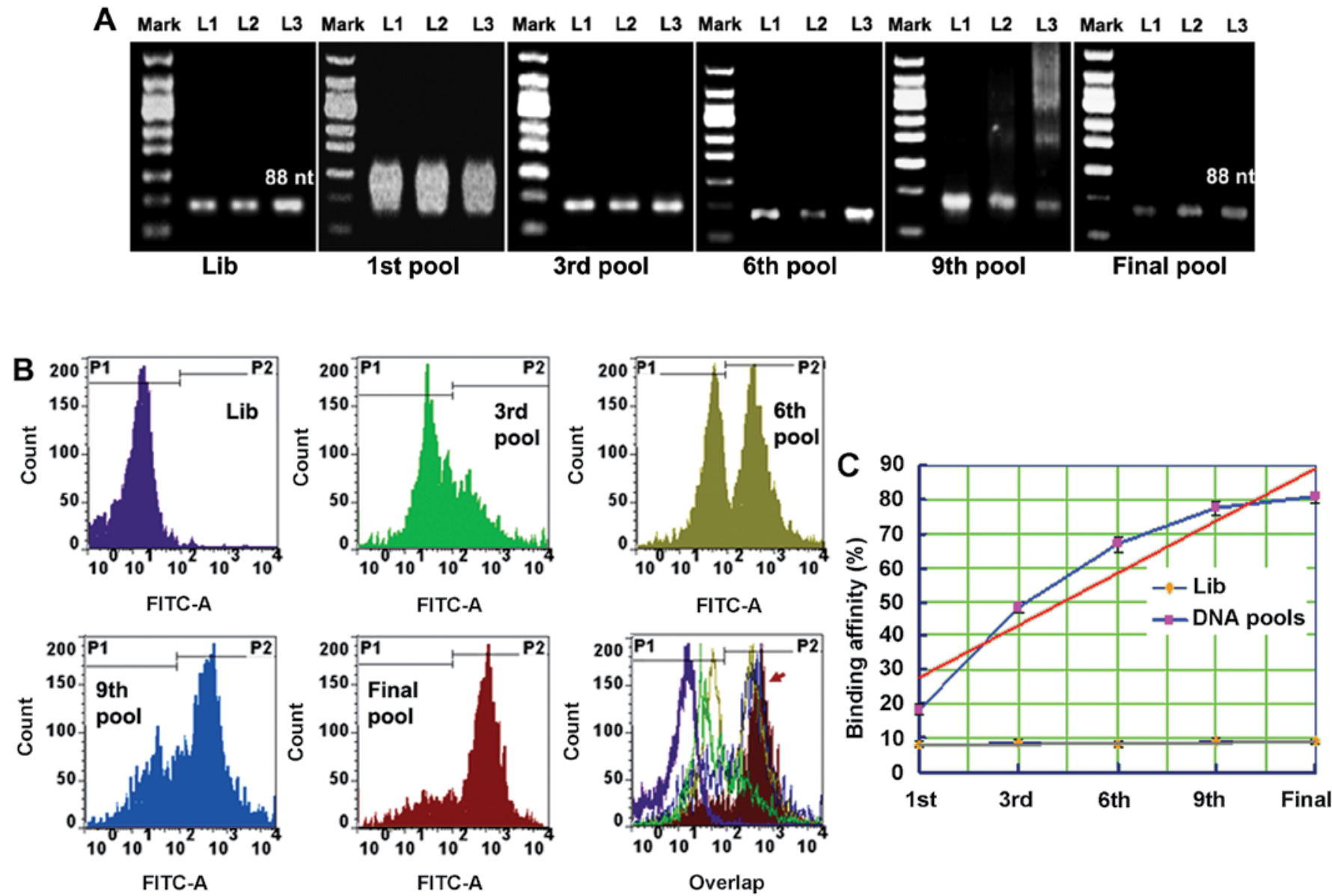

Figure 1. Evolution of gastric carcinoma cell-specific aptamers by cell-SELEX. Human gastric carcinoma AGS cells were used as target cells for initial selections and human normal gastric epithelial GES-1 cells as negative cells for counter selections. The selection procedure was monitored by gel electrophoresis and flow cytometric analysis. (A) Electrophoretograms of each selected pools during cell-SELEX. (B and C) Flow cytometry assessment of the enrichment of aptamer candidates specific to AGS cells. Increased rounds of selections denoted that the DNA probes with higher binding affinity to AGS cells were being enriched in the final pool (arrow). The concentration of FITC-labeled DNA probes used was 500 nM. Results are presented as means \pm standard error. FITC, fluorescein isothiocyanate; Lib, FITC-labeled unselected library ssDNA; L, electrophoretic lane.

Imaging of target cells stained with apatamer candidates. The specificity of the aptamer candidate cy-apt 20 in recognizing AGS cells was then visualized by fluorescence imaging. HepG2 and SW620 cells were used as controls. Prior to the imaging, culture cells were washed twice with washing buffer in flat-bottomed 6-well plates (Costar, Corning, NY, USA) and then incubated with $400 \mathrm{nM}$ of FITC-labeled cy-apt 20 in $200 \mu \mathrm{l}$ binding buffer on ice for $40 \mathrm{~min}$. After washing, the stained cells were viewed using an inverted fluorescence microscope (TE2000; Nikon) using the standard-FITC filter set (excitation at $490 \mathrm{~nm}$ and emission at $520 \mathrm{~nm}$ ). Images of the stained cells were captured with a DXM1200F digital camera (Nikon).

Data processing and statistical analysis. Fluorescence was determined by flow cytometry by counting 10,000 events/ sample. Data were read and processed by FlowJo software (version 7.6 for Windows; Tree Star, Ashland, OR, USA). Relative intensity of gel bands and fluorescence signals of stained tumor cells were quantified using ImageJ software (version 1.47 for Windows; NIH, Bethesda, MD, USA). Results were presented as means \pm standard error.

\section{Results}

Evolution of gastric carcinoma cell-specific aptamers by cell-SELEX. In the present study, the entire live cell-SELEX method was utilized to generate gastric cancer cell-specific aptamers. Human gastric carcinoma AGS cells were used as target cells for positive selections and human normal gastric epithelial GES-1 cells as negative cells for counter selections. To avoid loss of important cell surface molecules in the process of selection, cultured cells were collected by non-enzymatic cell dissociation solution. To ensure the exclusion of common or shared molecules, the amount of cells used for counter selections was maintained at $>5$-fold that for positive selections. The ssDNA pool collected after each round of selection was amplified by PCR, and the product was used to prepare ssDNA for the subsequent round of selection. The enrichment of the selection ssDNA pool through successive selection was monitored by agarose gel electrophoresis and flow cytometry. Electrophoresis band position (Fig. 1A) and an increase in the fluorescence intensity compared to the library ssDNA were indications of successful selection (Fig. 1B). Increasing rounds of selection denoted a steady increase in the fluores- 

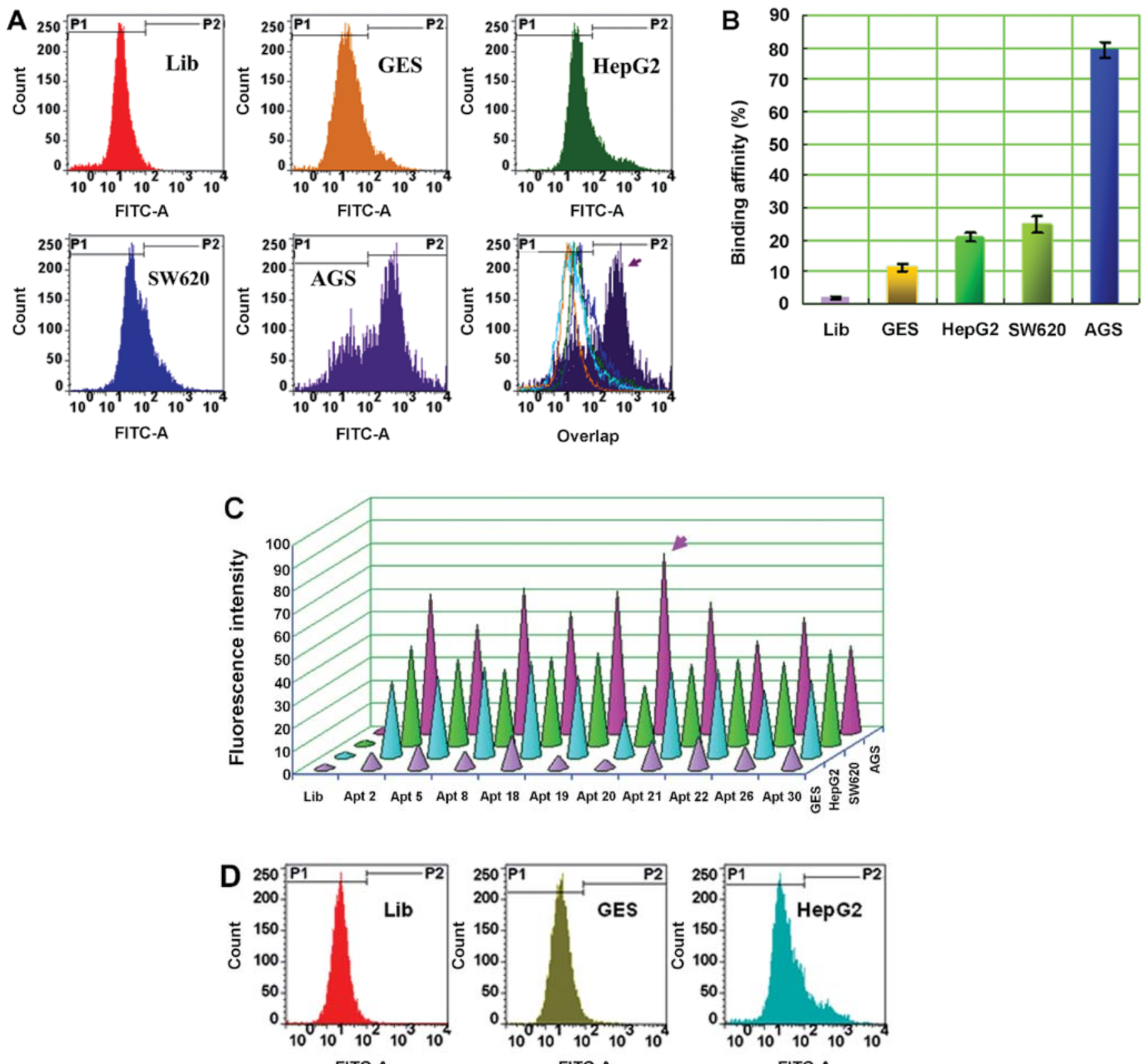

FITC-A

FITC-A
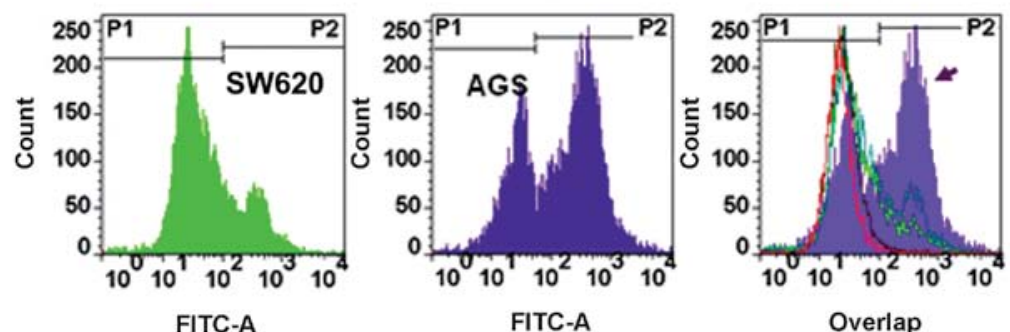

Figure 2. Identification of gastric cancer cell-specific DNA aptamers from the final pool. (A and B) The specificity of the final selected pool to AGS cells was analyzed using flow cytometry, and the concentration of FITC-labeled ssDNA used was $500 \mathrm{nM}$. The Lib to AGS cells and the final selected pool to GES, human hepatocellular carcinoma HepG2 cells and colon carcinoma SW620 cells were used as controls. Data are presented as means \pm standard error. The final selected pool was found to have much higher binding affinity to AGS than non-AGS cells (arrow). (C and D) By subsequent cloning and sequencing, 10 ssDNA sequences with high binding ability to AGS were identified from the selected aptamer candidates. Four of these ssDNA sequences were found have binding rates $>60 \%$ to AGS cells (data not shown), but only cy-apt 20 had $>70 \%$ binding rate to AGS cells with $<30 \%$ binding affinity to non-gastric carcinoma HepG 2 and SW620 cells (arrow notified). The concentration of FITC-labeled cy-apt 20 used was $400 \mathrm{nM}$. Data sre presented as means \pm standard error. Apt or cy-apt, identified ssDNA sequence.

cence intensity of the target cells, suggesting that the ssDNA sequences with a higher binding affinity to the target cells were being enriched (Fig. 1C).
Identification of a gastric cancer cell-specific DNA aptamer from the final pool. The final enriched ssDNA pool was analyzed by flow cytometry in comparison with GES, 

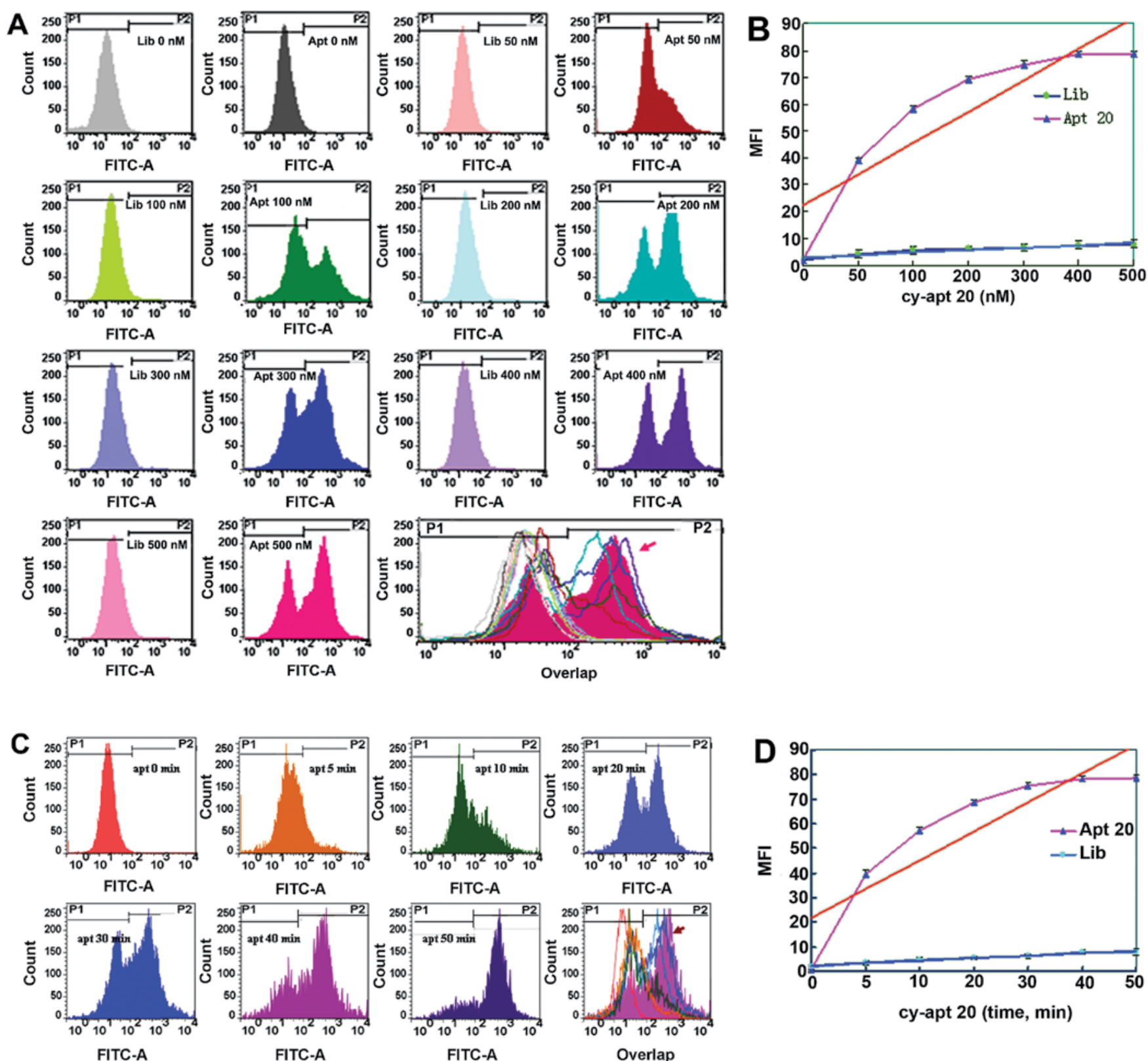

Figure 3. Characterization of the aptamer candidate cy-apt 20 in recognizing target cells. The tenable binding affinity and stability of cy-apt 20 to AGS cells was also assessed by flow cytometry in a dose- and time-dependent manner. Equivalent FITC-Lib ssDNA were used as controls and results were presented as means \pm standard error. (A and B) Increased binding rates were evident after 40 min of incubation with increasing concentrations of FITC-cy-apt 20, which peaked at $400 \mathrm{nM}$ (arrow). (C and D) Increased binding rates were observed by increasing the time length of incubation of AGS cells with $400 \mathrm{nM}$ of FITC-cy-apt 20 (arrow), which peaked at $40 \mathrm{~min}$.

HepG2 and SW620 cells. The results showed that the final enriched ssDNA pool had significantly higher binding affinity to AGS than non-AGS cells (Fig. 2A and B). The final selected ssDNA pool was cloned and the positive clones were sequenced. Sequence analysis identified 30 potential ssDNA sequences designated as cy-apt 01-30 as potential aptamer candidates specific to AGS cells (data not shown). A comparison of experiments were subsequently performed to confirm their target binding specificity towards AGS cells. Ten sequences had certain binding ability to AGS cells. Four of the 10 sequences had binding rates $>60 \%$ to AGS cells (Fig. 2C), with only cy-apt 20 exhibiting $>70 \%$ binding rate to AGS cells and $<30 \%$ binding affinity to non-gastric cancer cells (Fig. 2C and D).
Characterization of aptamer cy-apt 20 in recognizing target cells. The binding affinity and capacity of aptamer cy-apt 20 to AGS cells was also assessed by flow cytometry. Equivalent library ssDNA was used as controls under the same conditions as cy-apt 20. The concentrations of FITC-cy-apt 20 were initially varied from 0 to $500 \mathrm{nM}$, and then the time lengths of incubation varied from 0 to $50 \mathrm{~min}$. The data showed that the fluorescence intensity of AGS cells was steadily increased after 40 min of incubation with increasing concentrations of FITC-cy-apt 20 (Fig. 3A and B) and peaked at the concentration of $400 \mathrm{nM}$. The fluorescence intensity of AGS cells were also steadily elevated with increasing time length of incubation of AGS cells with FITC-cy-apt 20 and peaked at the time point of $40 \mathrm{~min}$ (Fig. 3C and D). 

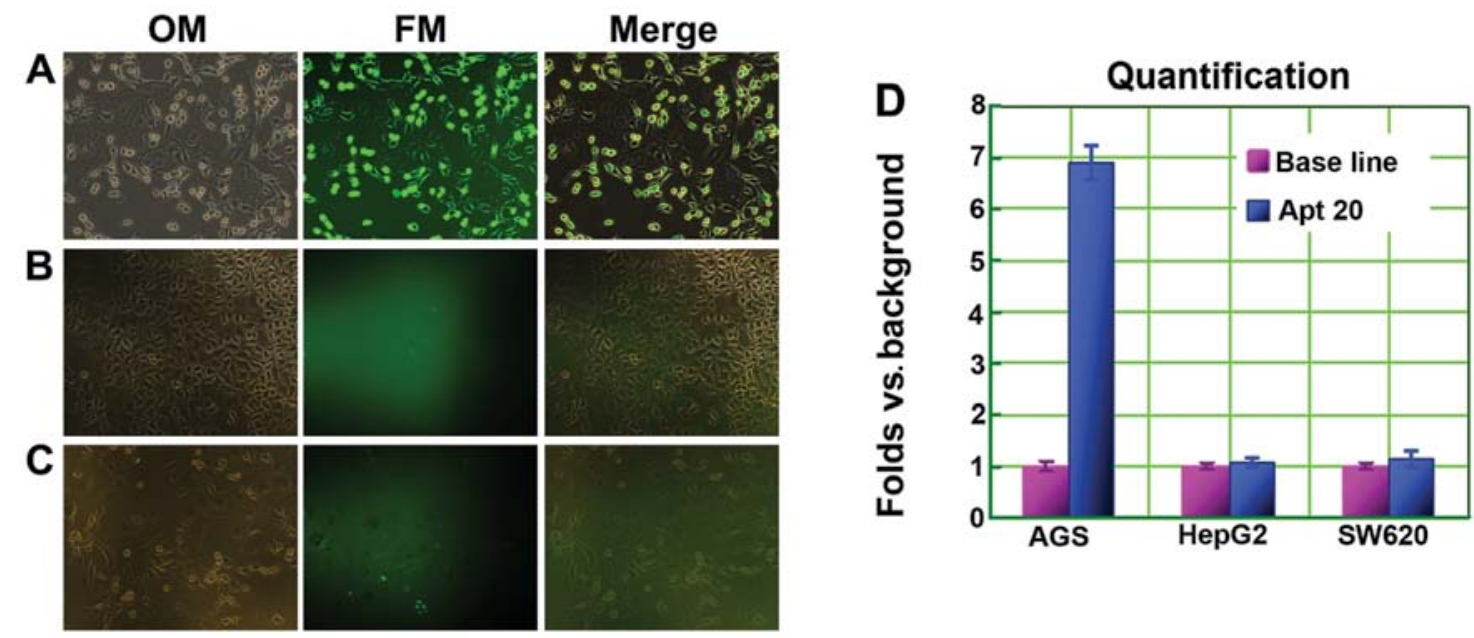

Figure 4. Fluorescence imaging of cancer cells using cy-apt 20. The specificity of the aptamer candidate cy-apt 20 in recognizing AGS cells was further visualized by fluorescence imaging, with HepG2 and SW620 cells being used as controls. All the three tumor cells were separately incubated with $400 \mathrm{nM}$ of FITC-labeled cy-apt 20 for $40 \mathrm{~min}$, and then observed with an inverted fluorescence microscope. (A) Images of AGS cells stained by FITC-labeled cy-apt 20 . Most AGS cells show certain extent of fluorescence intensity. Images of (B) HepG2 and (C) SW620 cells. Few viable HepG2 and SW620 cells exhibited detectable fluorescence. (D) Fluorescence signals of tumor cells relative to the background were quantified using NIH ImageJ software (version 1.47 for Windows) and results were presented as fold-changes vs. background. OM, optical microscope; FM, fluorescence microscope.

Fluorescence imaging of cancer cells using cy-apt 20. The specificity of aptamer cy-apt 20 in recognizing AGS cells was ascertained by fluorescence imaging, with HepG2 and SW620 cells being used as controls. The three tumor cells were separately incubated with $400 \mathrm{nM}$ of FITC-labeled cy-apt 20. After $40 \mathrm{~min}$ of incubation, the stained cells were viewed using an inverted fluorescence microscope. Most of the AGS cells were stained by FITC-labled cy-apt 20 (Fig. 4A and D). By contrast, few viable HepG2 (Fig. 4B and D) and SW620 (Fig. 4C and D) cells exhibited detectable fluorescence.

\section{Discussion}

The entire live cell-SELEX procedure has been proven to be a simple, but effective, reproducible and widely applicable procedure in generating high-affinity aptamers without previous knowledge of target molecules on tumor cells $(5,6)$. A large number of useful aptamers generated by this method are applied in the study of tumor biology, and even in the diagnosis and therapeutics of various types of cancer (12-21). The encouraging results obtained with aptamers combined with their intrinsic properties and the versatility of the live cell-SELEX procedure have led to the application of this technical method in the development of gastric cancer-specific DNA aptamers.

In the process of the culture cell-based selections, we used human gastric carcinoma AGS cells as target cells for positive selections and human normal gastric epithelial GES-1 cells as negative cells for counter selections. To avoid loss of important cell surface molecules in the process of selection, cultured cells were collected by non-enzymatic cell dissociation solution (10). To ensure the elimination of common or shared molecules on tumor and normal cells, the amount of cells used for counter selections was maintained at $>5$-fold that of positive selections $(9,10,22)$. Through 12 rounds of successive selections, as indicated by electrophoresis band position (Fig. 1A) and increased fluorescence intensity compared to the library ssDNA (Fig. 1B), a pool of ssDNA containing sequences with higher binding affinity to the target cells was enriched (Fig. 1C).

The final enriched ssDNA pool was subsequently confirmed by flow cytometric binding assays having high-binding capacity to the target cells, but with minimal recognition to the controls (Fig. 2A and B). By cloning and sequencing, we identified 30 ssDNA sequences termed cy-apt 01-30 as potential aptamer candidates specific to AGS cells (data not shown). A comparison of experiments subsequently identified 10 sequences from these candidates that bound to AGS cells, four of which had binding rates $>60 \%$ to AGS cells (Fig. 2C), whereas only cy-apt 20 had $>70 \%$ binding rate to AGS cells and $<30 \%$ binding affinity to non-gastric cancer cells (Fig. 2C and D). These results suggest that cy-apt 20 is a useful molecular tool for the recognition of gastric cancer cells.

The binding affinity and stability of cy-apt 20 in the recognition of AGS cells were assessed by flow cytometry in a dose- and time-dependent manner, since a molecular tool for detecting target cancer cells should possess tenable binding affinity and stability in addition to high specificity $(5,6,11,12)$. An equivalent library ssDNA was used as controls under the same conditions as those for cy-apt 20. The results show that the fluorescence intensity of AGS cells was steadily increased after $40 \mathrm{~min}$ of incubation with increasing concentrations of FITC-cy-apt 20 and peaked at the concentration of $400 \mathrm{nM}$ (Fig. 3A and B). Similarly, the fluorescence intensity of AGS cells was enhanced with the increasing time length of incubation with $400 \mathrm{nM}$ of FITC-cy-apt 20, which peaked at $40 \mathrm{~min}$ (Fig. 3C and D). The results suggest that targeting recognition can be established by using a minimal dose of cy-apt 20 that continued over a long period of time.

The feasibility of using aptamer cy-apt 20 for detecting gastric cancer cells was ascertained by fluorescence imaging, as an optimal biomarker-based diagnosis should be produced in direct, simplified and visualized ways $(1,2,4,23)$. The three tumor cells were separately incubated with $400 \mathrm{nM}$ of FITC-labeled cy-apt 20 for the comparisons made. After 
40 min of incubation, the stained cells were viewed using an inverted fluorescence microscope. The imaging showed that the majority of AGS cells were stained by FITC-labeled cy-apt 20 (Fig. 4A), whereas, few HepG2 cells (Fig. 4B) and SW620 cells (Fig. 4C) were stained by FITC-labeled cy-apt 20. The fluorescence intensity of AGS cells was $~ 7$-fold that of HepG2 and SW620 cells (Fig. 4D). The results suggest that cy-apt 20 is a useful molecular tool for detecting gastric cancer cells.

In summary, the results of the present study have demonstrated that the entire live cell-SELEX procedure was simple, but effective in generating cancer cell-specific aptamers, as previously reported (12-20). By using this method, we have identified a DNA aptamer termed cy-apt 20 with tenable binding affinity and stability to AGS cells. Additional investigations are required to determine the feasibility of using cy-apt 20 for detecting gastric cancer in vivo.

\section{Acknowledgements}

The present study was supported by a grant from the Nanjing Medical Science and Technology Development Foundation. We would like to thank the members of the Experiment Center of Basic Medicine of Nanjing Medical University, Jie Ling, Xian-Bo Zhu and Xiang Zhu, for valuable scientific discussions, cell culture and flow cytometric analysis. We also thank Professor Marie-Davis Du for her kind assistance in the writing of the manuscript.

\section{References}

1. Pietrantonio F, De Braud F, Da Prat V, et al: A review on biomarkers for prediction of treatment outcome in gastric cancer. Anticancer Res 33: 1257-1266, 2013.

2. Takahashi T, Saikawa Y and Kitagawa Y: Gastric cancer: current status of diagnosis and treatment. Cancers 5: 48-63, 2013.

3. Jemal A, Bray F, Center MM, et al: Global cancer statistics. CA Cancer J Clin 61: 69-90, 2011.

4. Yang S and Chung HC: Novel biomarker candidates for gastric cancer. Oncol Rep 19: 675-680, 2008.

5. Lassalle HP, Marchal S, Guillemin F, et al: Aptamers as remarkable diagnostic and therapeutic agents in cancer treatment Curr Drug Metab 13: 1130-1144, 2012.
6. Radom F, Jurek PM, Mazurek MP, et al: Aptamers: molecules of great potential. Biotechnol Adv 31: 1260-1274, 2013.

7. Hermann T and Patel DJ: Adaptive recognition by nucleic acid aptamers. Science 287: 820-825, 2000.

8. Tuerk $C$ and Gold L: Systematic evolution of ligands by exponential enrichment: RNA ligands to bacteriophage T4 DNA polymerase. Science 249: 505-510, 1990.

9. Stoltenburg R, Reinemann C and Strehlitz B: SELEX - a (r) evolutionary method to generate high-affinity nucleic acid ligands. Biomol Eng 24: 381-403, 2007.

10. Sefah K, Shangguan D, Xiong X, et al: Development of DNA aptamers using Cell-SELEX. Nat Protoc 5: 1169-1185, 2010.

11. Cerchia L and de Franciscis V: Targeting cancer cells with nucleic acid aptamers. Trends Biotechnol 28: 517-525, 2010.

12. Shangguan D, Li Y, Tang Z, et al: Aptamers evolved from live cells as effective molecular probes for cancer study. Proc Natl Acad Sci USA 103: 11838-11843, 2006.

13. Sefah K, Tang ZW, Shangguan DH, et al: Molecular recognition of acute myeloid leukemia using aptamers. Leukemia 23: 235-244, 2009.

14. Zhang K, Sefah K, Tang L, et al: A novel aptamer developed for breast cancer cell internalization. Chem Med Chem 7: 79-84, 2012.

15. Kunii T, Ogura S, Mie M and Kobatake E: Selection of DNA aptamers recognizing small cell lung cancer using living cellSELEX. Analyst 136: 1310-1312, 2011.

16. Sefah K, Meng L, Lopez-Colon D, et al: DNA aptamers as molecular probes for colorectal cancer study. PLoS One 5: e14269, 2010

17. Zhu G, Ye M, Donovan MJ, et al: Nucleic acid aptamers: an emerging frontier in cancer therapy. Chem Commun 48: 10472-10480, 2012.

18. Zhang Y, Chen Y,Han D, et al: Aptamers selected by cell-SELEX for application in cancer studies. Bioanalysis 2: 907-918, 2010.

19. Sefah K, Bae KM, Phillips JA, et al: Cell-based selection provides novel molecular probes for cancer stem cells. Int J Cancer 132: 2578-2588, 2013.

20. Zueva E, Rubio LI, Ducongé F and Tavitian B: Metastasisfocused cell-based SELEX generates aptamers inhibiting cell migration and invasion. Int J Cancer 128: 797-804, 2011.

21. Berezovski MV, Lechmann M, Musheev MU, et al: Aptamerfacilitated biomarker discovery (AptaBiD). J Am Chem Soc 130: 9137-9143, 2008.

22. Fang $X$ and Tan W: Aptamers generated from cell-SELEX for molecular medicine: a chemical biology approach. Acc Chem Res 43: 48-57, 2009.

23. Pavlou MP, Diamandis EP and Blasutig IM: The long journey of cancer biomarkers from the bench to the clinic. Clin Chem 59: 147-157, 2013. 